\title{
EFEITO DA INTENSIDADE DE DESBASTE NA QUALIDADE DA MADEIRA SERRADA DE Eucalyptus grandis
}

\author{
Rômulo Trevisan ${ }^{1}$, Clóvis Roberto Haselein ${ }^{2}$, Élio José Santini ${ }^{3}$, Paulo Renato Schneider ${ }^{3}$, \\ Leonel Freitas de Menezes ${ }^{4}$ \\ ${ }^{1}$ Eng. Florestal, M.Sc., Doutorando em Engenharia Florestal, UFSM, Santa Maria, RS, Brasil - trevisanr@smail.ufsm.br \\ ${ }^{2}$ Eng. Florestal, Ph.D., Depto. de Ciências Florestais, UFSM, Santa Maria, RS, Brasil - clovis.haselein @ smail.ufsm.br \\ ${ }^{3}$ Eng. Florestal, Dr., Depto. de Ciências Florestais, UFSM, Santa Maria, RS, Brasil - eliosantini@ smail.ufsm.br - paulors@ smail.ufsm.br \\ ${ }^{5}$ Eng. Florestal, International Business in Eucalyptus, Santa Maria, RS, Brasil - fmleonel@terra.com.br \\ Recebido para publicação: 26/10/2007 - Aceito para publicação: 05/04/2009
}

\begin{abstract}
Resumo
Este estudo teve como objetivo verificar o efeito da intensidade de desbaste na qualidade da madeira serrada de Eucalyptus grandis W. Hill ex Maiden aos 14 anos de idade. Para tanto, foi analisado um experimento de desbaste situado próximo ao litoral do Rio Grande do Sul, instalado no delineamento em blocos ao acaso, com quatro tratamentos e quatro repetições. As árvores foram selecionadas com base nos diâmetros dominantes e médios de cada tratamento. Após a derrubada, foi retirada a tora localizada entre as posições DAP e $25 \%$ da altura comercial, para a confecção de tábuas destinadas à avaliação do índice de rachaduras de topo e arqueamento. Os resultados mostraram que o maior espaço vital reduziu o índice de rachaduras de topo em tábuas de árvores dominantes e não apresentou tendência clara para as árvores médias. Com relação ao estrato amostrado, esse defeito foi maior nas árvores médias. $\mathrm{O}$ arqueamento das tábuas sofreu influência do desbaste nas árvores dominantes e médias, porém sem apresentar uma tendência definida com relação a sua intensidade. Na comparação desse defeito entre as árvores dominantes e médias não foi detectado diferença significativa.

Palavras-chave: Desdobro; defeitos; qualidade da madeira.
\end{abstract}

\begin{abstract}
Effect of thinning intensity in the sawn wood quality of Eucalyptus grandis. This study was undertaken to determine the effect of thinning intensity in the sawn wood quality of 14 years old Eucalyptus grandis W. Hill ex Maiden. There was analyzed a thinning experiment, located in the northern coast of Rio Grande do Sul installed in randomized blocks, with four treatments and four repetitions. The trees were selected on the basis of the dominant and average diameter at breast height (DBH) of each treatment. After cutting down, there were selected logs located between DBH and $25 \%$ of the commercial height, for evaluations of end splits and board spring. The results indicated that thinning intensity reduced end splits of boards of dominant trees and did not present a clear trend for the average ones. In relation to the sampled stratum, this defect was higher in average trees. The board spring was influenced by thinning in both dominant and average trees, however, without presenting a clear trend with its intensity. The comparison of this defect between dominant and average trees showed no significance.

Keywords: Sawn wood; defects; wood quality.
\end{abstract}

\section{INTRODUÇÃO}

Atualmente, o aumento da oferta de matéria-prima proveniente de reflorestamentos tem despertado o mercado consumidor a preocupar-se com a qualidade da madeira. Os progressos alcançados, principalmente em produtividade florestal, são cada vez mais significativos e, nos últimos anos, tem-se notado um crescente interesse dos setores florestal e industrial na busca de matéria-prima homogênea e de qualidade, a fim de maximizar seu potencial de uso e, consequentemente, minimizar os problemas no processo produtivo.

O manejo da floresta pode ser uma contribuição importante para a melhoria da qualidade e produtividade dos povoamentos florestais. Nesse sentido, o desbaste é um dos mais importantes 
tratamentos silviculturais, por proporcionar espaço para o desenvolvimento da copa e do sistema radicular e, consequentemente, melhor aproveitamento de luz, nutrientes e água, dentre outras características (GALVÃO, 1992; SCHNEIDER, 2002; SOARES et al., 2003).

Assim, o planejamento do desbaste, segundo Schneider (2002), tem por objetivos manipular a competição entre as árvores, evitar consequências da competição excessiva, evitar a permanência de indivíduos com má formação de fuste e direcionar o potencial produtivo do sítio para as árvores de maior valor comercial.

Apesar de ser reconhecida como matéria-prima para energia e outros produtos, Rocha; Tomaselli (2002) e Lopes (2003) destacam que ainda existem certas restrições com respeito à madeira serrada do gênero Eucalyptus. Os principais problemas ocorrem quando as espécies são provenientes de povoamentos com rápido crescimento, devido a sua propensão a rachaduras e ao empenamento, o que diminui a aceitação e prejudica a utilização da madeira para fins mais nobres (LIMA, 2005).

Conforme Jankowsky (1995), Del Menezzi (1999) e Vermaas (2000), esses problemas são os principais fatores de redução de rendimento industrial, sendo próprios e inerentes ao uso de florestas jovens, nas quais os níveis de tensões de crescimento manifestam-se de forma mais proeminente que em florestas maduras.

De acordo com Dinwoodie (1966), as tensões de crescimento existentes na madeira são definidas como forças que se desenvolvem no interior dos troncos de árvores vivas, sendo características intrínsecas ao crescimento natural das árvores (JACOBS, 1945). Essas tensões são determinadas a partir da medição da alteração nos comprimentos das peças de madeira, da dimensão das rachaduras, tanto em toras quanto em tábuas, e da flecha dos empenamentos (LISBÔA, 1993; PONCE, 1995; DEL MENEZZI, 1999; SILVA, 2000; LIMA et al., 2004).

O nível e a distribuição dessas tensões em árvores não são bem conhecidos, mas há suspeitas de que estejam relacionados a parâmetros de crescimento que podem ser influenciados através do manejo silvicultural (FERRAND, 1983; WILKINS; KITAHARA, 1991; MALAN; HOON, 1992; MIRANDA; NAHUZ, 1999), fatores genéticos, idade, dimensões da tora, inclinação do fuste e sistema de desdobro utilizado (OPIE et al., 1984; ZOBEL; JETT, 1995; ROCHA; TOMASELLI, 2002; LIMA et al., 2004).

Este trabalho foi desenvolvido com árvores de diâmetro dominante e médio, com o objetivo de verificar o efeito da intervenção silvicultural de desbaste na qualidade da madeira serrada de Eucalyptus grandis $\mathrm{W}$. Hill ex Maiden aos 14 anos de idade.

\section{MATERIAL E MÉTODO}

\section{Localização e caracterização da área de estudo}

A área experimental localiza-se na Empresa Flosul Indústria e Comércio de Madeiras Ltda., nas coordenadas geográficas de $50^{\circ} 30^{\prime}$ de longitude oeste e $30^{\circ} 08^{\prime}$ de latitude sul, no município de Capivari do Sul, litoral norte do estado do Rio Grande do Sul.

A precipitação pluviométrica média anual para a cidade de Viamão, próxima à região de estudo, fica em torno de $1500 \mathrm{~mm}$, e a temperatura média anual é de aproximadamente $19,0{ }^{\circ} \mathrm{C}$ (NIMER, 1989).

\section{Obtenção dos dados}

As sementes utilizadas na produção das mudas para implantação do experimento são da espécie Eucalyptus grandis Hill ex Maiden, procedência Botucatu (PSC - COFFS HARBOUR). Essas mudas foram produzidas no viveiro florestal da própria empresa, no ano de 1990. Dessa forma, os dados para o presente estudo foram obtidos de parcelas permanentes, em experimento de desbaste estruturado em blocos ao acaso, com quatro repetições para cada tratamento e implantado com espaçamento inicial de $3,0 \times 1,7 \mathrm{~m}$.

Os tratamentos foram caracterizados como: T0 (testemunha, densidade completa), T1 (desbaste até 30\% da área basal da testemunha), T2 (desbaste até 60\% da área basal da testemunha) e T3 (desbaste até $90 \%$ da área basal da testemunha). O experimento foi medido até o décimo quarto ano de idade, e os desbastes foram realizados de acordo com plano de redução da área basal. A tabela 1 apresenta os tratamentos analisados e as condições atuais do experimento.

A amostragem das árvores utilizadas foi realizada com base no diâmetro à altura do peito (DAP). Foram coletadas 16 árvores dominantes, com diâmetro médio de $37,1 \pm 2,1 \mathrm{~cm}$, e 16 árvores médias, com diâmetro médio de $27,0 \pm 5,5 \mathrm{~cm}$, totalizando 32 árvores. 
Tabela 1. Tratamentos de desbaste analisados aos 14 anos de idade.

Table 1. Thinning treatments analyzed at 14 years of age.

\begin{tabular}{lcccccc}
\hline \multirow{2}{*}{ Tratamentos } & $\begin{array}{c}\text { Desbastes } \\
\text { executados }\end{array}$ & Bloco 1 & Bloco 2 & Bloco 3 & Bloco 4 & \multirow{2}{*}{ Média } \\
\cline { 2 - 5 } & 0 & 1925 & 1675 & 1400 & 1550 & 1638 \\
T0 & 1 & 1125 & 1150 & 1175 & 1150 & 1150 \\
T1 & 3 & 589 & 589 & 589 & 589 & 589 \\
T2 & 6 & 190 & 185 & 196 & 196 & 192 \\
T3 & & &
\end{tabular}

\section{Determinação dos defeitos da madeira serrada}

Para a determinação dos defeitos da madeira serrada, foram selecionadas a primeira tora da árvore dominante e média de cada tratamento, localizada entre a posição do DAP e $25 \%$ da altura comercial, que corresponde ao diâmetro mínimo de $13 \mathrm{~cm}$ para a entrada de toras na serraria.

Após a derrubada das árvores e seccionamento das toras, elas foram imediatamente transportadas até a serraria da empresa, onde o sistema de desdobro tangencial foi executado, inicialmente, pelo corte das duas costaneiras, com auxílio da serra de fita dupla. Posteriormente, utilizando a serra circular múltipla, transformaram-se os semiblocos em tábuas. As tábuas, com dimensões comerciais médias de 25 x 155,0 × $3300 \mathrm{~mm}$, foram avaliadas com respeito a rachaduras de topo e arqueamento, principais defeitos desenvolvidos por ocasião do desdobro.

\section{Rachaduras de topo das tábuas}

$\mathrm{O}$ índice de rachaduras de topo das tábuas foi determinado através do somatório dos comprimentos das rachaduras presentes nas duas faces das tábuas. $\mathrm{O}$ valor percentual do índice de rachaduras foi obtido pela razão entre a soma dos comprimentos das rachaduras pelo comprimento total da tábua (Equação 1).

$$
\mathrm{IR}_{\mathrm{tb}}=\left[\frac{\sum \mathrm{LR}}{2 \mathrm{Lr}}\right] 100
$$

Em que: $\mathrm{IR}_{\mathrm{tb}}=$ índice de rachadura de topo da tábua, em \%;

$\sum \mathrm{L}_{\mathrm{R}}=$ somatório dos comprimentos das rachaduras de topo nas duas faces da tábua, em $\mathrm{cm}$;

$\mathrm{Lr}=$ comprimento total da tábua, em $\mathrm{cm}$.

Arqueamento das tábuas

Entende-se por arqueamento a curvatura ao longo do comprimento da peça de madeira num plano paralelo à face, sendo determinado pela equação 2 .

$$
\mathrm{AR}=\left[\frac{\mathrm{X}}{\mathrm{Lr}}\right] 100
$$

Em que: $\mathrm{AR}=$ arqueamento da tábua, $\mathrm{em} \mathrm{mm} / \mathrm{m}$;

$\mathrm{X}=$ concavidade, $\mathrm{em} \mathrm{mm}$;

$\mathrm{Lr}=$ comprimento total da tábua, em $\mathrm{m}$.

\section{Análise estatística dos dados}

Os dados médios de rachaduras de topo e do arqueamento das tábuas produzidas a partir das árvores dominantes e médias em função da intensidade de desbaste foram submetidos à análise de variância, utilizando-se o pacote estatístico Statgraphics, e no caso de rejeição da hipótese de igualdade de médias entre tratamentos, ao teste de médias (Least Significant Difference - LSD, $\alpha=5 \%$ ).

\section{RESULTADOS E DISCUSSÃO}

\section{Rachaduras de topo das tábuas}

Os resultados encontrados para o índice de rachaduras de topo das tábuas, tanto para as árvores dominantes (Prob. $>\mathrm{F}=0,0099$ ) quanto para as médias (Prob. $>\mathrm{F}=0,0001$ ), aos 14 anos de idade, evidenciam que houve influência significativa da intensidade de desbaste em nível de $1 \%$ de 
probabilidade. Na tabela 2 é apresentado o teste de comparação de médias das rachaduras de topo das tábuas em relação às intensidades dessa intervenção silvicultural.

Tabela 2. Comparação de médias do parâmetro índice rachaduras de topo das tábuas das árvores dominantes e médias em função da intensidade de desbaste.

Table 2. Comparison of means of the parameter end splits index of the boards of the dominant and average trees in function of thinning intensity.

\begin{tabular}{lccc}
\hline & Árvores Dominantes & \multicolumn{2}{c}{ Árvores Médias } \\
\hline Tratamentos & $\begin{array}{c}\text { Índice de rachadura de } \\
\text { topo das tábuas }(\%)\end{array}$ & Tratamentos & $\begin{array}{c}\text { Índice de rachadura de } \\
\text { topo das tábuas }(\%)\end{array}$ \\
\hline $\mathrm{T} 1$ & $18,7 \mathrm{a}$ & $\mathrm{T} 2$ & $41,1 \mathrm{a}$ \\
$\mathrm{T} 0$ & $13,7 \mathrm{~b}$ & $\mathrm{~T} 1$ & $20,8 \mathrm{~b}$ \\
$\mathrm{~T} 2$ & $12,7 \mathrm{~b}$ & $\mathrm{~T} 0$ & $15,1 \mathrm{~b}$ \\
$\mathrm{~T} 3$ & $10,2 \mathrm{~b}$ & $\mathrm{~T} 3$ & $14,1 \mathrm{~b}$ \\
\hline
\end{tabular}

Médias seguidas pela mesma letra em cada coluna não diferem significativamente entre si em nível de 5\% de probabilidade.

Para as árvores dominantes, a comparação de médias (Tabela 2) mostra que o tratamento 1 apresentou maior índice de rachaduras de topo das tábuas $(18,7 \%)$, diferindo estatisticamente dos demais tratamentos. A análise mostra também que a menor média desse defeito $(10,2 \%)$ ocorreu no tratamento 3 , o qual apresentou maior espaço vital.

Esses resultados estão de acordo com Wilkins; Kitahara (1991), os quais verificaram que intervenções silviculturais de desbastes efetuados em uma população de Eucalyptus grandis, aos 12,5 anos de idade, aumentaram a taxa de crescimento das árvores e diminuíram a tensão de crescimento, que, segundo Ferrand (1983) e Lisbôa (1993), é a causa principal do aparecimento de rachaduras na madeira. As mesmas conclusões foram descritas por Miranda; Nahuz (1999), afirmando que o maior espaçamento de plantio reduziu a percentagem de rachaduras de topo das tábuas e, consequentemente, a perda de material serrado.

Ferrand (1983), analisando o efeito dos desbastes nas tensões de crescimento em Eucalyptus delegatensis e Eucalyptus nitens, verificou que intervenções moderadas não influenciaram essa característica. Entretanto, desbastes intensos e precoces reduziram as tensões

A comparação de médias para as árvores médias (Tabela 2) evidencia que o maior valor percentual de rachaduras de topo das tábuas ocorreu no tratamento 2 , com valor de $41,1 \%$, sendo diferente estatisticamente dos demais tratamentos. É interessante observar, a partir desses resultados, a inexistência de interação (positiva ou negativa) significativa entre as intensidades de desbaste e a porcentagem de rachaduras de topo das tábuas das árvores médias.

Da mesma forma, Malan; Hoon (1992), Lima et al. (2000) e Lima (2005) observaram que desbastes gradativos não tiveram uma tendência sistemática de reduzir ou aumentar o aparecimento de rachaduras de topo das tábuas de Eucalyptus grandis, concluindo que o desenvolvimento desse defeito está ligado, principalmente, a fatores genéticos (LIMA et al., 2004).

Zobel; Jett (1995) relatam que para Eucalyptus grandis e Eucalyptus globulus, devido à grande variação das rachaduras de árvore para árvore, é possível a seleção e clonagem de genótipos com menor propensão a esse efeito.

Comparando esse índice entre as árvores dominantes e médias, verifica-se que existe diferença significativa em nível $\alpha=5 \%$ de probabilidade e Prob. $>\mathrm{F}=0,0021$. Na tabela 3 , é apresentado o teste de comparação de médias das rachaduras de topo das tábuas em relação aos estratos amostrados, evidenciando que o maior valor percentual de rachaduras de topo das tábuas ocorre nas árvores médias, com valor de $21,9 \%$, sendo diferente estatisticamente das árvores dominantes que, em média, apresentaram um decréscimo de $8,0 \%$ desse defeito.

Tabela 3. Comparação de médias do parâmetro rachadura de topo das tábuas das árvores dominantes e médias.

Table 3. Comparison of means of the parameter end splits of the dominant and average trees.

\begin{tabular}{lc}
\hline Estrato amostrado & Índice de rachadura de topo das tábuas $(\%)$ \\
\hline Dominante & $13,9 \mathrm{a}$ \\
Médio & $21,9 \mathrm{~b}$ \\
\hline Médias seguidas pela mesma letra em cada coluna não diferem significativamente entre si em \\
nível de 5\% de probabilidade.
\end{tabular}




\section{Arqueamento}

O resumo da análise de variância para arqueamento das tábuas evidencia que tanto as árvores dominantes (Prob. $>\mathrm{F}=0,0004$ ) quanto as médias (Prob. $>\mathrm{F}=0,0001$ ) foram influenciadas significativamente pela intensidade de desbaste, em nível $\alpha=5 \%$ de probabilidade.

A comparação de médias para as árvores dominantes, apresentada na tabela 4, mostra que a intervenção de desbaste proporcionou a incidência de arqueamento das tábuas, porém sem tendência com a intensidade. Isso pode ser observado a partir da constatação de que os maiores índices desse defeito ocorreram nos tratamentos 1, 2 e 3, onde foi empregada essa intervenção silvicultural, diferindo estatisticamente quando comparados com a testemunha, sem desbaste. Para as árvores médias verificouse que não houve uma tendência clara do efeito do desbaste no arqueamento das tábuas.

Tabela 4. Comparação de médias do parâmetro arqueamento das tábuas das árvores dominantes e médias em função da intensidade de desbaste.

Table 4. Comparison of means of the parameter board spring of the dominant and average trees in function of the thinning intensity.

\begin{tabular}{lccc}
\hline & Árvores dominantes & \multicolumn{2}{c}{ Árvores médias } \\
\hline Tratamentos & Arqueamento $(\mathbf{m m} / \mathbf{m})$ & Tratamentos & Arqueamento $(\mathbf{m m} / \mathbf{m})$ \\
\hline T1 & $4,25 \mathrm{a}$ & $\mathrm{T} 3$ & $6,45 \mathrm{a}$ \\
T2 & $3,91 \mathrm{a}$ & $\mathrm{T} 0$ & $4,15 \mathrm{~b}$ \\
T3 & $3,70 \mathrm{a}$ & $\mathrm{T} 2$ & $2,79 \mathrm{~b}$ \\
T0 & $1,93 \mathrm{~b}$ & $\mathrm{~T} 1$ & $2,63 \mathrm{~b}$ \\
\hline
\end{tabular}

Médias seguidas pela mesma letra em cada coluna não diferem significativamente entre si em nível de 5\% de probabilidade.

A tabela 4 mostra ainda que, para as árvores dominantes, todas as médias de arqueamento das tábuas em função das intensidades de desbaste estão de acordo com a Norma para Classificação de Madeira Serrada de Folhosas (INSTITUTO BRASILEIRO DE DESENVOLVIMENTO FLORESTAL, 1983), que regulamenta um máximo de defeito igual a $5 \mathrm{~mm} / \mathrm{m}$ em relação ao comprimento total da tábua. Já para as árvores médias, nota-se que apenas o tratamento 3, com valor médio de arqueamento igual a $6,5 \mathrm{~mm} / \mathrm{m}$, não está de acordo com essa Norma. A comparação desse defeito entre árvores do estrato médio e dominante não foi significativa em nível $\alpha=5 \%$ de probabilidade, com médias de 4,0 $\mathrm{mm} / \mathrm{m}$ e $3,4 \mathrm{~mm} / \mathrm{m}$, respectivamente.

Um aspecto importante que deve ser considerado na análise do arqueamento das tábuas refere-se ao sistema de desdobro utilizado. De forma geral, sabe-se que o desdobro tangencial minimiza os efeitos dessa característica quando comparado, por exemplo, com o desdobro radial. Rocha; Tomaselli (2002), ao desdobrarem toras de Eucalyptus grandis, evidenciaram um decréscimo médio de 7,63 $\mathrm{mm} / \mathrm{m}$ quando utilizado o desdobro tangencial.

\section{CONCLUSÕES} conclusões:

Os resultados obtidos neste estudo para Eucalyptus grandis aos 14 anos permitem as seguintes

- Para as árvores dominantes, o maior espaço vital reduziu o índice de rachaduras de topo das tábuas, enquanto que nas árvores médias não houve tendência significativa com a intensidade dessa intervenção. Com relação ao estrato amostrado, esse defeito mostrou-se superior nas árvores médias.

- O arqueamento das tábuas das árvores dominantes e médias foi influenciado pelo desbaste, porém, sem tendência com a intensidade dessa intervenção. Já a comparação desse defeito entre as árvores dominantes e médias não foi significativa.

- Com base nas características tecnológicas das árvores dominantes e médias, pode-se afirmar que o planejamento e emprego da intervenção silvicultural de desbaste no povoamento florestal deverá considerar a utilização final da madeira.

\section{AGRADECIMENTOS}

Os autores agradecem a Empresa Flosul Indústria e Comércio de Madeiras Ltda. pela colaboração na realização deste trabalho. 


\section{REFERÊNCIAS}

DEL MENEZZI, C. H. S. Utilização de um método combinado de desdobro e secagem para a produção de madeira serrada de Eucalyptus grandis Hill ex Maiden e Eucalyptus cloeziana F. Muell. 87 p. Dissertação (Mestrado em Ciência Florestal) - Escola Superior de Agricultura "Luiz de Queiroz", Piracicaba, 1999.

DINWOODIE, J. M. Growth stresses in timber: a review of literature. Forestry, London, v. 39, n. 2, p. 162-170, 1966.

FERRAND, J. C. Growth stresses and silviculture of eucalypts. Australian Forest Research, Canberra, v. 13, n. 1, p. 75-81, 1983.

GALVÃO, P. M. Aspectos fundamentais do uso e da comercialização da madeira de pinus no Brasil. In: SIMPÓSIO FLORESTAL DO RIO GRANDE DO SUL - TECNOLOGIA DA MADEIRA, 2. 1992, Esteio. Anais... Santa Maria: CEPEF/FATEC, 1992. p. 30-39.

INSTITUTO BRASILEIRO DE DESENVOLVIMENTO FLORESTAL (IBDF). Norma para classificação de madeira serrada de folhosas. Brasília, DF: Brasiliana, 1983. 67 p.

JACOBS, M. R. The growth stresses of wood stems. BULL. Commonwealth Forestry Burean, Canberra, n. 28, p. 1-67, 1945.

JANKOWSKY, I. P. Equipamentos e processos para a secagem de madeiras. In: SEMINÁRIO INTERNACIONAL DE UTILIZAÇÃO DA MADEIRA DE EUCALIPTO PARA SERRARIA, 1995, São Paulo. Anais... São Paulo: IPEF/IPT/IUFRO/LCF/ESALQ/USP, 1995, p. 109-118.

LIMA, I. L. Influência do desbaste e da adubação na qualidade da madeira serrada de Eucalyptus grandis Hill ex Maiden. 161 p. Tese (Doutorado em Engenharia Florestal) - Escola Superior de Agricultura “Luiz de Queiroz”, Piracicaba, 2005.

LIMA, I. L.; GARCIA, J. N.; NOGUEIRA, M. C. S. Influência do desbaste nas tensões de crescimento de Eucalyptus grandis Hill ex Maiden. Scientia Forestalis, Piracicaba, n. 58, p. 111-125, 2000.

LIMA, J. T.; TRUGILHO, P. F.; ROSADO, S. C. da. S.; CRUZ, C. R. da. Deformações residuais longitudinais decorrentes de tensões de crescimento em eucaliptos e suas associações com outras propriedades. Revista Árvore, Viçosa, MG, v. 28, n. 1, p. 107-116, 2004.

LISBÔA, C. D. J. Estudo das tensões de crescimento em toras de Eucalyptus grandis Hill ex Maiden. 275 p. Tese (Doutorado em Ciências Florestais) - Universidade Federal do Paraná, Curitiba, 1993.

LOPES, M. C. Agrupamento de árvores matrizes de Eucalyptus grandis em função das variáveis dendrométricas e das características tecnológicas da madeira. 93 p. Dissertação (Mestrado em Engenharia Florestal) - Universidade Federal de Santa Maria, Santa Maria, 2003.

MALAN, F. S.; HOON, M. Effect of initial spacing and thinning on some wood properties of Eucalyptus grandis. South African Forestry Journal, Pretoria, n. 163, p. 13-20, 1992.

MIRANDA, M. J. A. C.; NAHUZ, M. A. R. Estudo da influência do espaçamento de plantio de Eucalyptus saligna Smith nos índices de rachamento após o desdobro e após a secagem. Scientia Forestalis, Piracicaba, n. 55, p. 107-116, 1999.

NIMER, E. Climatologia do Brasil. 2 ed. Rio de Janeiro: IBGE, Departamento de Recursos Naturais e Estudos Ambientais, 1989, 422 p.

OPIE, J. E.; CURTIN, R. A.; INCOLL, W. D. Stand management. In: HILLIS, W.; BROWN, A. G. Eucaliptos for wood production. Melbourne: CSIRO, 1984. 434 p. 
PONCE, H. R. Madeira serrada de Eucalipto: desafios e perspectivas. In: SEMINÁRIO INTERNACIONAL DE UTILIZAÇÃO DA MADEIRA DE EUCALIPTO PARA SERRARIA, 1., 1995, São Paulo. Anais... São Paulo: IPT, 1995. p. 50-58.

ROCHA, M. P.; TOMASELLI, I. Efeito do modelo de desdobro na qualidade da madeira serrada de Eucalyptus grandis e Eucalyptus dunnii. Cerne, Lavras, v. 8, n. 2, p. 70-83, 2002.

SCHNEIDER, P. R. Manejo Florestal: planejamento da produção florestal. Santa Maria: UFSM, 2002. $492 \mathrm{p}$.

SILVA, J. C. Características da madeira de eucalipto. Revista da Madeira, Curitiba, v. 9, n. 52, 2000. p. 30-38.

SOARES, T. S.; SILVA, M. L. da; GAMA, J. R. V.; CARVALHO, R. M. M. A.; VALE, R. S. do. Avaliação econômica de plantações de Eucalipto submetidas a desbaste. Revista Árvore, Viçosa, MG, v. 27 , n. 4, p. 481-486, 2003.

VERMAAS, H. F. A. Review of drying technology for young fast-grown eucalypts. In: THE FUTURE OF Eucalyptus FOR WOOD PRODUCTS, Launceston, 2000. Proceedings... Launceston: IUFRO, 2000. p. 193-203.

WILKINS, A. P.; KITAHARA, R. Silvicultural treatments and associated growth rates, growth strains and wood properties in 12,5-year-old Eucalyptus grandis. Australian Forestry, Yarralumla, v. 54, n. 1/2, p. 99-104, 1991.

ZOBEL, B. J.; JETT, J. B. Genetics of Wood Production. Syracuse: Springer-Verlag, 1995. 